\title{
Analytical Techniques for Decision Making on Information Security for Big Data Breaches
}

\author{
Aiiad Albeshri* and Vijey Thayananthan ${ }^{\dagger}$ \\ Department of Computer Science \\ Faculty of Computing and Information Technology \\ King Abdulaziz University \\ Jeddah 21589, Kingdom of Saudi Arabia \\ *aaalbeshri@kau.edu.sa \\ †vthayanathan@kau.edu.sa
}

Published 9 February 2018

\begin{abstract}
In the big data processes, management and analytics are primary areas where we can introduce the decision making on information security to mitigate the big data breaches. According to the growing number of online systems and big data handling, mitigating the big data breaches is the serious problem during the processing period which needs to be monitored using appropriate technique. The goal of this research is to prevent the big data breaches using correct decision making based on information security concepts such as access control with authentication which depend on the management policies. The analytical approach of information security solution can also be useful for securing the big data infrastructure and key management that improve the big data breaches. As an analytical method, information security which focuses on detecting and securing the big data breaches is considered with access control. Here, we have introduced the multi-priority model influenced with the network calculus and access control which monitors the breaches during the big data processing. In the results and analysis, we can provide a graph which shows the monitoring improvement for decision making during the mitigation of big data breaches.
\end{abstract}

Keywords: Analytical techniques; big-data breaches; decision making; information security; service rate.

\section{Introduction}

This section is a key area for introducing the current situation of the big data breaches and cyber intelligence. The cost of the big data breaches and how organizations manage the appropriate security solution to mitigate these issues are increasing. The big data analytical techniques depend on the set of advanced technologies designed to corporate with key properties of the big data. They are such as

\footnotetext{
$\dagger$ Corresponding author.

This is an Open Access article published by World Scientific Publishing Company. It is distributed under the terms of the Creative Commons Attribution 4.0 (CC-BY) License. Further distribution of this work is permitted, provided the original work is properly cited.
} 
large volume, different varieties, and appropriate velocity. In the analytical techniques, ${ }^{1-4}$ we can employ the computational mathematics, statistical approach, machine learning, etc. In this research, a delay which causes the breach problem is analyzed through the network calculus that allows us to perform the services calculations considered as one of the big data analytical methods. ${ }^{5}$

According to the research report. ${ }^{6}$ identifying big data breaches takes a long time (many days or months). Some cases such as sensitive data take more than years. Thus, the big data analytical approach might help us to reduce most of the problems related to big data security breaches. Big data security breaches cost an organization millions of dollars and multiple incidents. Protecting against the threat of big data breach is not easy in popular and government organizations because access controlling is not monitored properly. Further, multiple unauthorized accessing situations are increased.

The provenance of the big data should be corresponding to where each phase of the process takes in the big data analytical techniques. Finding the exact point of the security incident is not easy during the processing. Thus, we have introduced the multi-priority model which incorporates with network calculus to find the breach monitoring service requests.

From the references mentioned in above paragraphs, we can study the definitions of big data such as high volume, high velocity, high variety, etc. Some of them are already considered for improving security in many applications, but they are not focused clearly on making decisions to avoid the big data breaches. When service providers try to distribute the big data to various places, decision making should be dealt efficiently with many factors they are information security, priorities, etc.

The volume of the big data refers to the data sizes which could be in terabytes or petabytes or zettabytes. In big data analytical techniques, processing is the important procedure which models and analyses the properties of the big data based on the actual size of the big data might be considered with time complexity.

A variety of big data refers to the conditions of the data structures which are three categories; they are structured, unstructured and semi-structured. In the analytical technique of big data, these conditions play an important role because processing needs statistical analysis.

Velocity refers to the rate of big data during the processing which could be useful when service providers are modeling and analyzing the big data security breaches. For instance, modern organizations handle more than one million transactions within in an hour. Although velocity and transactions per hour are varying with many factors, the velocity of text, audio and video formats at each phase can be analyzed using appropriate big data analytical technique.

These days, Internet and ICT developers focus on efficient decision making because it reduces the overall cost rather than spending more time on the design and development new security solutions. We have investigated and identified many big data analytical techniques which are convertible to the designing system used for decision making. Here decision making on information security increases the 
warnings of the breaches and provides the number of security facilities as extra guards during the processing of big data. They are anti-theft policies updated dynamically, automation of access control, etc. but they have to be validated through the appropriate analytical technique which incorporates with the big data.

Contributions which deserve to mitigate the big data breaches based on the efficient decision making are given below.

- Traditional analytical techniques are not capable of managing big data breaches efficiently. Therefore, a multi-priority model with appropriate information security tool which provides us encryption and decryption to secure the access control is considered. Although access control security already exists with different computational approaches, we prefer to use network calculus as a novel contribution.

- With the proposed model, delay performance will be considered and calculated in three different priorities which help us to improve the decision making of big data breaches.

- In the research analysis, we provide the service rate and delay calculations based on network calculus which dominates to improve the request rates. Analyzing the properties of big data through our model enables us to enhance the accuracy of the decision making.

The rest of the paper is organized as follows. Section 2 focuses on related work includes analytical technique, mitigation, and decision making. In Sec. 3, we provide problem statement used in this research through the system descriptions and model involved with the access control. Section 4 explains the proposed scheme with selected parameters and priorities. Implementation and necessary results obtained from the selected priorities are organized in Sec. 5. Also, this section analyses that improvement of mitigation and decision making on information security. Enhancement of preventing big data breaches is possible through the appropriate analytical technique of decision making. In Sec. 6; overall conclusions are written based on the theoretical analysis and results.

\section{The Related Work}

According to Refs. 7 and 8, authors studied the big data security breaches helped to construct the secure big data architecture with the latest technology that includes the mitigation approach of security solutions. Here, mitigating big data breaches need correct security measurements on time. Although arrival and departure time of the big data is recorded by the access control, quality and quantity of big data need to be monitored using appropriate security tools. In their approach, information security was the top priority to create the big data architecture that supports to develop the appropriate analytical technique of big data. The most of the current working environment uses Internet and information technology but mitigating big data breaches, and analytical techniques of decision making are not standardized properly. Regarding the big data security issues, they have focused on 
big data security analysis flow based on Hadoop, which is a big data analytics software platform.

Many security issues mentioned in Ref. 9 are very useful to protect the big data in all applications. Most of the security issues considered for the big data protections apply to all organizations, but their legal policies may not be allowed or valid. In this study, authors have used the own attributes that have more power than the existing attributes. They also pointed that securing the valuable contents of big data was not easy, but they studied the relevant attributes and applied for protecting big data during the processing.

According to Ref. 10, information security policy which are management, technology, economy, and culture is verified with analytic hierarchy process (AHP) considered as the methodology of decision making. Also, information security components such as confidentiality, integrity, and availability enhanced the security measurements with the use of AHP model. Authors claimed that AHP model helped policymakers of the big data breaches to deliver the appropriate decisions. To increase the quality of the decision making, AHP shows a robust. Therefore, AHP model can be used for a larger scope of research.

As mentioned in Ref. 11, decision making introduced for multi-criteria using hesitant fuzzy sets (HFSs) is proposed. Although four different approaches mentioned in this article, HFS provides the big data to improve the breaching through the appropriate decision making. In the first, HFS is involved with two hesitant fuzzy numbers. Second, the generalized hesitant Hausdorff distance is developed to enhance the decision-making approach. Third, based on the proposed comparison method and distance, decision making is verified. Finally, pragmatism and effectiveness of the proposed approaches considered with appropriate examples. Further, authors claimed that the proposed methods do not require complicated computation procedures.

According to Ref. 12, big data plays an important role to support the decision making and helps us to improve the problem-solving in decision making. Despite the big data breaches, decision-making process such as information systems, data repositories, formal modeling, and analysis of decisions depend on the information security. Here, authors have defined the key components of the big data which allows us to design the integrated model of decision making using business intelligence and decision support systems.

According to Ref. 13, game theory helps decision makers to investigate the multiperson strategic decision making through the appropriate mathematical tools and models. As a special class of games, security games and their solutions allow researchers to design and develop the new decision making approaches when we have interaction problems between malicious attackers and defenders.

Reference 14 provides detailed information on game theory and model which are important to data providers who take the decision when data provider is affected by several factors such as privacy. Here, authors have claimed that game theory provides a formal approach to model situations where a group of agents has to choose optimum 
actions considering the mutual effects of other agents' decisions. Further, they have proposed a game model to analyze the interactions among the active users (data providers, collectors, etc.). In this model, the data collector could employ privacypreserving data publishing (PPDP) techniques to protect data providers' privacy.

According to Ref. 15, the economic theory of privacy which include economic debate of current situations has been analyzed to protect the personal data. The economics of the personal data and privacy are very prominent concepts for improving decision making when many users are involved. Reference 16 explains the contact theoretic approach which allows data collector to make a rational decision when data owners are struggling with their data.

According to Ref. 17, authors have collected and studied the decision-making methods, types of decision and necessary algorithms for decision making as a literature review since 2015. Further, they explained about the priority derivation methods to analyze the decision making introduced in the pairwise comparison matrix (PCM). Despite many algorithms used in the decision making, the multiple criteria decision-making (MCDM) and stochastic preference analysis (SPA) methods are popular. Since previous researchers have introduced the missing judgments in the PCM, missing judgments processing models could be used to analyze the decision problems in the big data environment.

In the clustering algorithms, methods of MCDM plays a vital role because the evaluation of clustering algorithms involves typically multiple criteria. Regarding all performance criteria used in the financial risk analysis, Ref. 18 provides a new evaluation approach based on MCDM to assess the quality of clustering algorithm. Although all algorithms do not provide the best performance according to the data size, repeated-bisection approach outperforms when selected cluster algorithm is used for specific data. Hence, proposed approach could be extended to analyze the decisions on big data breaching.

According to Ref. 19, proposed approach allows decision makers to resolve and decide disagreements among MCDM methods. In this approach, ensemble technique involved with decision stump which is a simple one-level decision tree improves the decisions. Based on the performance of the selected data, some MCDM methods are used to evaluate the classification algorithms. Regarding the big data, proposed approach may resolve conflicting MCDM rankings generated in different situations such as monitoring breaches. The weights of MCDM methods could be useful for the new challenges of the future research because they play an important role to analyze the decision problems using Spearman's rank correlation coefficient equations.

Reference 20 explains the analytic hierarchy process (AHP) which is one of the MCDM methods, and AHP supports to decision makers in many applications including big data breaching. Despite the three main types of decision problems (ranking, sorting, and choice), the ranking is considered with AHP. It subsequently discusses the impact of the proposed model depended on a flexible and efficient cosine maximization (CM) based on similarity measure for a priority vector derivation from a PCM. Since researchers know that the optimization model produces a unique 
solution with the $\mathrm{CM}$, we can apply the reliable priority vector to improve the decision problems depending on the optimization.

In the decision making considered with PCMs, cardinal and ordinal inconsistencies are very popular research topics. ${ }^{21}$ Regarding the consistencies, authors have proposed a Hadamard product induced bias matrix (HPIBM) model. Further, HPIBM enhances matrix consistency when we use priority derivation method in the decision-making problem which includes a decision on big data breaching. Further, numerical examples allow researchers to understand the illustration of the proposed model for improving the inconsistency ratios. Instead of considering other approaches ${ }^{21}$ HPIBM can be used for identification and adjustment of both cardinal and ordinal inconsistencies.

\subsection{Decision-making tool and big data analytical techniques}

Big data handlers such as broadcasting and astronomy industries show how computational method and big data support and provide the decision making. As shown in Fig. 1, decision-making tool based on information security for big data breaches can be developed with the efficient computational approach and big data analytical techniques. Further, information security goals improve not only the breaching accuracy of the decision making but also the efficiency of managing realtime decision-making tool and decision support system.

These points, which descriptive, predictive, and prescriptive provide deep knowledge to improve the decision-making tools. Despite these points, the mitigation of big data breach can be used to set the following information security goals:

- To evaluate the performance of information security algorithms which deal with policies used within the organization.

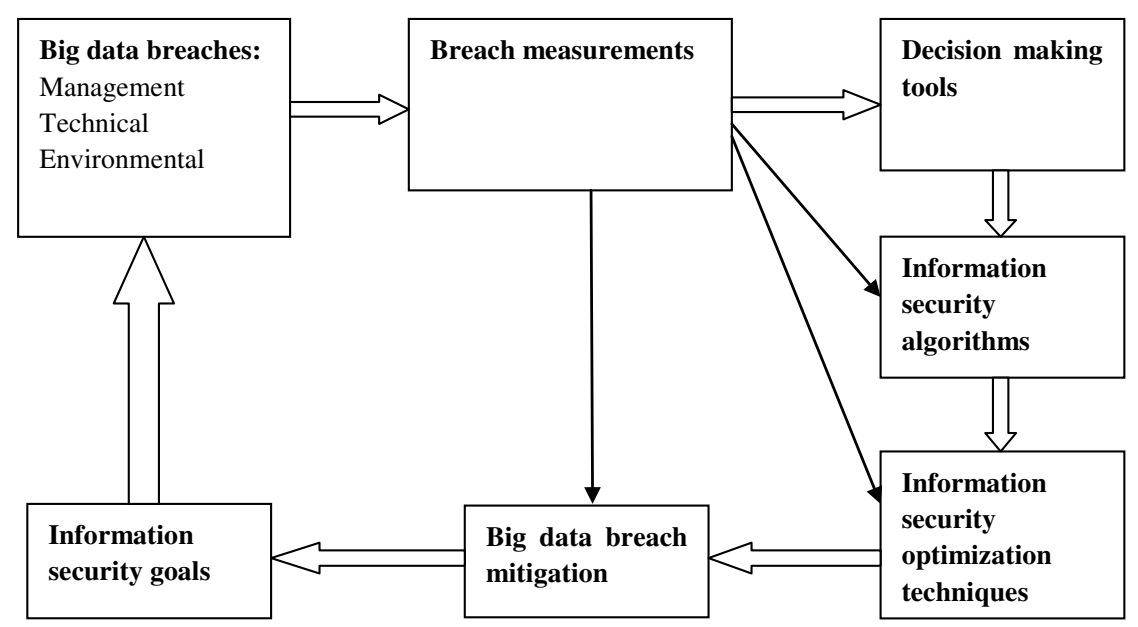

Fig. 1. Decision-making tool based on information security for big data breaches. 
- To optimize protection level of technical capacity which focuses on information security optimization techniques.

- To manage the quality of the big data from the public and private sectors where monitoring, storing and securing are important and depend on the improvement of the organizational security level.

- To add the contribution related to the type of the big data with existing decision making, information security, and current technology. Here, all types should be identified within the organization process.

- To calibrate the systems used for big data breaches. Further, technical verifications should be apprehended by the organizations on behalf of service providers.

- To justify the storage and transmission costs related to the information security.

According to Refs. 22-24, big data processing that depends on high-volume, highvelocity, and high-variety provides decision making to enhance the security through the access control. In the recent papers, researchers focus on the security issues of data and programming framework which depend on the correct security tools, but the use of access control provides many advantages. They are mitigation of big data security breaches during the big data processing. These programming framework related to security monitoring resources reduce the overall cost when service providers utilize the appropriate security during big data processing. In this approach, authors only focus on big data security and programming framework based on minimizing dynamic resources using necessary security algorithm with the information security concepts. ${ }^{25-27}$ These proposed approaches will improve not only the big data security but also it enhances the security of services influenced by the types of the big data.

Analytics as a service (AaaS) is one of the requirements when users handle the big data in the cloud environments. ${ }^{28,29}$ In an AaaS insight framework, users and service providers found the problems of big data breaches monitored from data delivery and management to data usages. Till developing appropriate prevention techniques, big data breaches will be the biggest problem within the cloud environment. If we prevent these breaches and other threats such intrusions and network security issues ${ }^{30,31}$ future data centers and organizations involved with big data will have a comprehensive cloud-based big data strategy.

\subsection{Mitigation of big data breaches}

When modern adversaries try to use different types of attack to breach the big data security, existing security solution may not be possible for mitigating big data breaches during the big data processing. ${ }^{32,33}$ Some cases, existing solutions may be used to resolve the breaches issues from various perspectives. In the big data processing, all phases of the processing have to be considered, but full mitigation may not be possible. However, a big data platform is a complete system with multiple activities of the users and service providers. Thus, the system may not tolerate any big data security breaches and lose all types of data during the processing. Although 


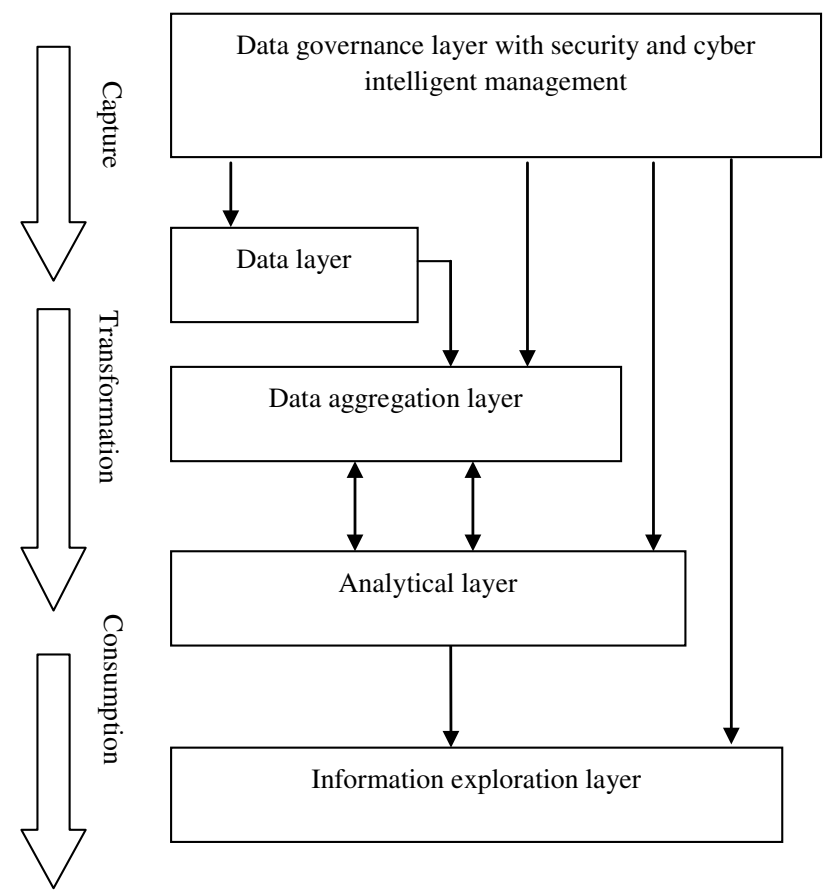

Fig. 2. Big data analytical architecture for mitigating security breaches.

we have studied various forms of big data analytical techniques which ${ }^{34}$ are associated with potential applications of big data such as patients records in big organizations, breaching cannot be completely removed. Figure 2 elaborates the details of the architecture which mitigate and minimize the breaching problems.

In this paper, monitoring breach service at access control is considered as an example of an intelligent approach which can be derived from the big data analytical technique. Despite the decision making based on information security, access control depends on the analytical techniques based on the current security tools.

Data management looks after the managerial benefits depending on the automatic updates which are the intelligent services. To deliver the correct decision making based on the cyber intelligence, big data analytical techniques such as processes and supporting technologies help us to analyze the service rate and delay. We have selected five benefits of big data analytical techniques as shown in Table 1. To enhance each benefit of analytical technique, we can choose the correct mitigation and possible decision making based on information security.

\subsection{Decision making based on cyber intelligence}

Benefits of big data analytical techniques are many, but we have selected five important techniques which can be enhanced through appropriate intelligent systems. However, decision making based on the cyber intelligence which is the specific policy 
Table 1. Improvement of benefits used in big data analytics.

\begin{tabular}{|c|c|c|}
\hline $\begin{array}{l}\text { Benefits of the big } \\
\text { data analytics }\end{array}$ & Mitigation of big data breaches & Possible decision making \\
\hline IT infrastructures & $\begin{array}{l}\text { Correct security tools and key } \\
\text { management }\end{array}$ & Automation \\
\hline Operations & Efficient security algorithms & Self-monitoring \\
\hline Organizational & Valid date legal agreements & Self-renewal procedures \\
\hline Managerial & Upto date legal policies & Automatic updates \\
\hline Strategic & Future compatible technology & $\begin{array}{l}\text { Predictions of the behavior } \\
\quad \& \text { forecasting }\end{array}$ \\
\hline
\end{tabular}

of the information security depends on the intelligent systems. As mentioned in Table 1, decision making based on information security, efficient computational approaches and cyber intelligence also can be used to boost the benefits of the big data analytical techniques. ${ }^{35}$ For instance, facial recognition can be one of the future compatible technologies which not only empower the mitigation but also help the proposed model to acquire cyber intelligence. As people become older with strange behavior, their faces acquire more character which can be identified through the facial recognition. Further, big data analytical techniques can be enhanced through the decision making based on cyber intelligence which influences the human behavior such as facial changes and movement patterns within the organizations. ${ }^{36}$ The big data collected from various sources such as video cameras in any organizations can be considered to analyze the cyber intelligence of the information security issues. Regarding the big data collections, for instance, some health organizations use the smart algorithms which can collect demographic information about patients who make the hospital appointments. We are constantly striving to improve our decision making and intelligent service through the smart algorithms which depend on the appropriate information security tools and big data analytical approach.

Employing network calculus in the multi-priority model characterizes the network performance which allows us to make an accurate decision. Sametime, it also allows us to improve the delays during the big data processing. In the big data analytical technique, processing can be modeled and analyzed when we monitor the arrival traffic of the big data at each phase of the processing. Access control monitors the delays and service request rates at the arrival traffic.

\section{Problem Statement}

According to the investigations, future design faces the following key problems that influence the big data breaches:

- When untrusted sources handle the uploading, capturing, storing and extracting structured and unstructured big data, users and service providers address many problems such as unauthorized access, big data breaches, etc. 
- Inexperienced skill sets also create the big data breaches when users or service providers perform data integration, analysis, transformation, and visualization. Because of these problems, delivering the right contents of big data to the right location may be delayed.

- Poor supervising, managing and controlling big data under comprehensive policy and governance guidelines.

\subsection{System description}

As shown in Fig. 3, big data analytical technique can be organized with three security solutions and access control which provide the breach monitoring services.

The combination of selected algorithms studied from information security theory will be considered as an appropriate method that provides the necessary functionalities to handle the above problems. Here, correct security tools like efficient cryptography techniques will be possible with the necessary modifications that fit the specifications of big data. Reliable key management techniques that not only secure the useful facilities such as storage and transmission in the cloud system but also protect the infrastructure. To implement above approaches, we develop a theoretical model that includes the appropriate skills with necessary protocols and algorithms. Both users and service providers will be able to employ the theoretical model whenever they want and wherever they need. Despite these skills, the including heterogeneous platform within the theoretical model is another challenging problem because when the big data file is transferring from machine to machine or system to system, there is no guarantee that access control provides the sustainable protections to that transferred file. The likelihood is that big data could be exposed to potential big data breaches within the organizations.

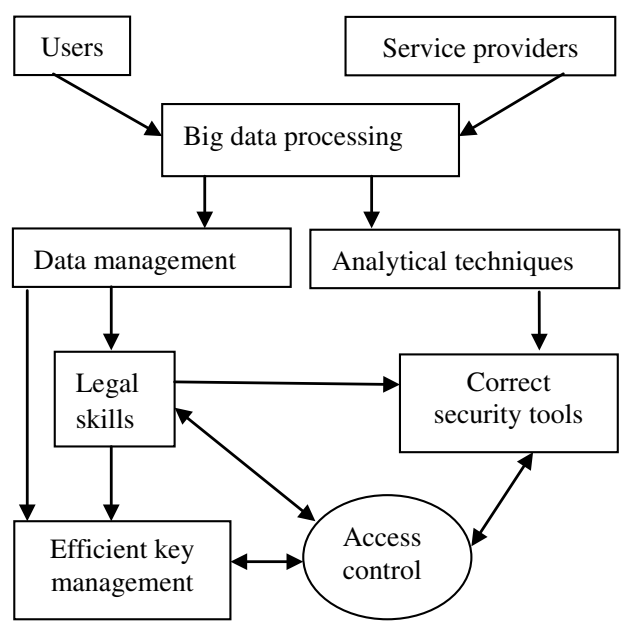

Fig. 3. Big data breaching with analytical technique. 


\subsection{Big data breaching model}

Figure 4 shows the recommended model for big data breaching. Based on the priorities, processing buffer collects the big data and calculate the service rate for the breach monitoring requests per second. In this model, we can employ any security tools like standard encryption and decryption algorithms. Here, we have introduced the network calculus which allows us to calculate the service requests during the big data processing. Despite the efficient service requests, decision making based on information security depends on the service rate.

Although access control is not monitored properly, many external problems easily damage the big data processing which reduces the monitoring capacities of the big data breaches. Other problems caused by cloud services also may create the big data breaches. Although service providers look after all the services, third parties can create the big data breaches when they utilize the cloud services. Accidental damages, fault resources, and other human errors also are the problems that create the big data breaches. Here, big data that contains all confidential information should be protected from following problems that occur in a cloud environment. They are data loss, accidental and purpose deletion, insufficient diligence, account hijacking, etc.

Constant parameters are determined according to the weight of the service which depends on the priority services, size of the queue, etc. In this paper, we have considered a constant parameter which is a reference $\left(b_{C}\right)$ known as fixed priority obtained from Ref. 37. Other parameters (low $\left(b_{L}\right)$, medium $\left(b_{M}\right)$ and high $\left(b_{H}\right)$ priorities) are also assumed as completely arbitrary. Reference is the fixed constant parameter which provides the correct and best decision determined from the real practical situations.

\subsection{Elaboration of network calculus algorithm}

Network calculus is a set of mathematical rules and results which can be used for computing performance of man-made systems such as communication network,

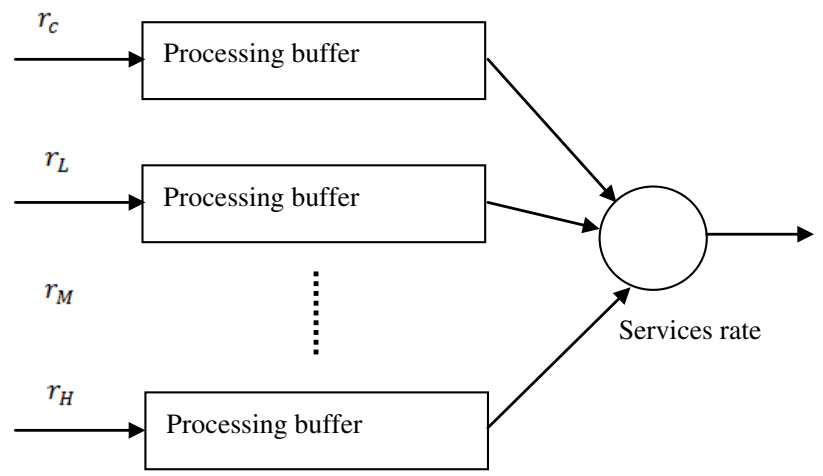

Fig. 4. Multi-priority access control model for big data communication. 
computer network applications. In our paper, we have introduced the network calculus which allows us to calculate the delays versus service rates during the big data processing. Further, we have chosen network calculus algorithm as the steps of the multi-priority model because it provides the best solution for breach control. Network calculus algorithm can be formed in different modes according to the applications and their performance analysis such as delay, link capacity, rate, traffic, etc. In recent results of systems' performance, network calculus use more min-plus algebra than max-plus algebra procedures as algorithms. ${ }^{37-39}$ Further, following definitions employed within network calculus algorithm are used to analyze the network performance which helps us to improve the time and/or accuracy of problem solving in decision making. ${ }^{40,41}$

Definition 1 (Flow Delay). Assume data flow with input A crosses the proposed model and results in the output $A^{\prime}$. The delay for a data unit arriving at time $t$ and bounded delay $(\tau)$ is given as $D(t)=\inf \left\{\tau \geq 0 \mid A(t) \leq A^{\prime}(t+\tau)\right\}$.

Definition 2 ((min, + )-Operations). The main (min, + )- algebraic operations used in the network calculus for functions $(f, g)$ and server $(s)$ are considered as below

Aggregation: $(f+g)(t)=f(t)+g(t)$

Convolution: $(f \otimes g)(t)=\inf _{0 \leq s \leq t}\{f(t-s)+g(s)\}$

Definition 3 (Sub-additive function). Let $f$ be a function used in the network calculus algorithm. Then $f$ is sub-additive if and only if $f(t+s) \leq f(t)+f(s)$ for all $s, t \geq 0$.

\section{Proposed Scheme}

Here, we propose a multi-priority approach as a theoretical model which monitor the behavior of the big data, minimize the breaches and determine the appropriate decision making. The solutions based on the information security and behavior depends on many factors such as service rate, delay, etc. which allow us to mitigate the big data breaches. In this proposed scheme, service performance calculations depend on the priorities which are high, medium and low. The big data analytical technique needs these priorities to determine the decision making, mitigate the big data breaches and cyber intelligence. First, we investigate the $R_{H}$, which is rate with high priority as below.

$$
R_{H}=R
$$

In (4.1), $R$ is the minimum service rate of the big data when the model is handling the big data with which we calculate the worst-case performance rate during the processing.

$$
T_{H}=\frac{L_{M a x}}{R_{H}} .
$$


Using (4.2), the latency of the access control during the processing of big data analytical approach can be obtained. Here, $L_{\text {Max }}$ is the maximum request length of the service considered by the big data when priorities lower than the selected.

$$
R_{M}=R-r_{H}-r_{C} .
$$

As (4.3), $R_{M}$ is the rate for the medium priorities can be calculated using appropriate rates $r_{H}$ and $r_{C}$. Here, these rates are considered as high and constant which is the fixed and set for performance comparisons, respectively.

$$
T_{M}=\frac{L_{M a x}+b_{H}+b_{C}}{R_{M}} .
$$

Burst tolerances $b_{H}$ and $b_{C}$ are the parameters used in (4.4) set by network calculus theory.

$$
R_{L}=R-r_{M}-r_{H}-r_{C}
$$

In (4.5), $R_{L}$ calculates the rate for lower priorities. Parameters $r_{M}$ and $b_{M}$ are rate and burst tolerance of the services created by the big data.

$$
T_{L}=\frac{b_{M}+b_{H}+b_{C}}{R_{L}} .
$$

The latency $\left(T_{L}\right)$ which represents delay with lower priorities can be calculated using (4.6). All latency calculations based on the delay of the services provides the QoS which is one of the big data analytical techniques to mitigate the big data breaches.

Breach monitoring services are like requests during the big data processing. Here, some requests for the services, delays based on the priorities determine the security of big data breaches.

\subsection{Analytical model}

There are many analytical models awaiting to which we can consider from different properties of big data and specific applications. Here, we have chosen network calculus as an algorithm of the multi-priority model which provides the best solution for breach control. The combination of these approaches can be implemented in big data analytical technique.

\subsection{Access control approach}

Access control of big data security breaches refers to ways of authorization which may depend on correct security tools, securing efficient key management and legal skill sets. Unfortunately, we cannot implement the access control as a sole secure solution to this breaching problem. Access privileges provide users and service providers the unlimited authorizations which must be legalized using up to date policies and valid agreements. These legal procedures can be maintained through the secured skill sets approved by the organizations. 


\section{Analysis of Big Data Breaches}

Big data breaches can be spotted when the unreasonable delay occurs during the processing which influences the properties of the big data. Further, we have elaborated one of the properties and some scenarios. Thus, the value is selected. It refers to levels of volume analyzed through the multi-priority model. In this analysis, velocity not only affects the flow rate but variability also influences to vary the flow rates of service requests. Variability depends on the veracity represented by the unreliable source of big data.

\subsection{Analysis of results}

In this analysis, three priorities are considered as in Fig. 5 where we only focused on the delay comparisons with the service rates. Delay bounds help service providers and users to make possible decisions which mitigate the big data breaching in different environments. Here, the results are obtained using Matlab which allow us to validate the proposed model with the necessary graphs as in Fig. 5. We set up a simulation scenario with three priorities they are high, medium and low as we mentioned in the proposed model. Here, all priorities and reference values are assumed as weights depending on the services and size of the queue, etc. In the horizontal axis, service rates are set as follows: $R \in[6000,8500]$ requests/second $(\mathrm{r} / \mathrm{s})$. Although all rates (reference $\left(r_{C}\right)$, low $\left(r_{L}\right)$, medium $\left(r_{M}\right)$ and high $\left(r_{H}\right)$ ) are fixed as $1500 \mathrm{r} / \mathrm{s}$, delays depend on the values of the priorities which are the average values of the random weights.

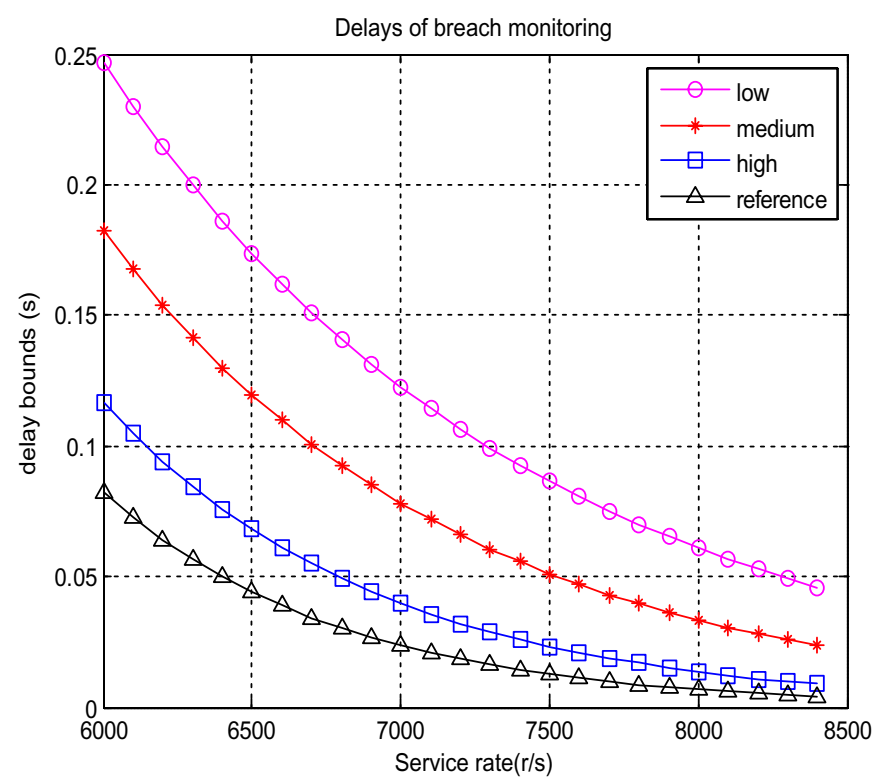

Fig. 5. Delay bounds for breach monitoring services. 
In this analysis, the result shows the random period of the big data where we can calculate the service request rate with delays. When monitoring service is with high priority, the delay is very close to the reference which is set from a real experiment. Therefore, request services are in high priority; we can improve the solution of big data security breaches.

According to the definitions of big data, size is the only one main parameter highlighting to implement the big data analytical technique. In this analysis, access control monitors the service rate of the big data based on the size and other parameters. Velocity and variety are also important to analyze the big data analytical techniques which provide the security solutions to mitigate the big data breaches, but they have been used to determine the request rate of the big data.

\subsection{Selected scenarios for big data breaches}

Big data can be categorized according to the type of organizations and their potential activities of the applications which may be real-time processing, storing, etc. of big data. For instance, hours of recorded video data, millions of patients profiles and their medical histories, millions of bank information, etc. can be considered as categories. Few of these categories are selected as scenarios.

Big data of bank information: Although many security techniques and surveillance are available, breaches are continuing. Using proposed model, technically we can reduce the big data breaching because our hybrid actions not only detect the technical aspects of the big data breaches through the appropriate analytical techniques but also identify the managerial and administrative aspects of the breaches through the cyber intelligence. Here, cyber incident reports in the repository, storage area, etc. and breaching situations during the big data processing can be analyzed through the access control.

Big data of video and its analytics: Speed of the vehicles are monitored through the video cameras which produce the millions of big data with different varieties. Here, the functions of the video cameras such us filming without clear zooming and deleting considered as big data breaches. These types of video analytics can also be mitigated through our proposed model.

\subsection{Implication of decision making for big data breaches}

Through state of the art and another theoretical background, the implication of decision making depends on the situations which may be around the big data storage and during the processing. These situations may be protected by the big data users, but if these situations are controlled by the service providers with inaccurate decision making, breaching situation will increase. Although the decision making procedure is automated using our analytical approach, policies, algorithms, etc. of information security should be powerful with current threats which create the breaching environments. To improve the decision making based on the situation, appropriate 
Table 2. Intelligence and analysis for decision making from big data to impact (see Ref. 42).

\begin{tabular}{|c|c|c|c|}
\hline & Business and market intelligence & $\begin{array}{l}\text { Modern science \& } \\
\text { technology }\end{array}$ & $\begin{array}{l}\text { Security issues for } \\
\text { public safety }\end{array}$ \\
\hline Application & $\begin{array}{l}\text { - Selected systems } \\
\text { - Business media } \\
\text { - Customer care } \\
\text { - Social channel, etc. }\end{array}$ & $\begin{array}{l}\text { - Legacy systems } \\
\text { - Innovation } \\
\text { - Smart techniques } \\
\text { - Hypothesis testing } \\
\text { - Knowledge }\end{array}$ & $\begin{array}{l}\text { - Cybersecurity } \\
\text { - Breaches } \\
\text { - Cyberterrorism } \\
\text { - Information leak } \\
\text { - Intelligence } \\
\text { - Crime }\end{array}$ \\
\hline Data & $\begin{array}{l}\text { - Service providers \& } \\
\text { care facilities } \\
\text { - Users information } \\
\text { - Users' handling }\end{array}$ & $\begin{array}{l}\text { - Channel quality } \\
\text { - Sensors data } \\
\text { - Network traffic } \\
\text { - Instruments' signals }\end{array}$ & $\begin{array}{l}\text { - Legal data } \\
\text { - Rumors } \\
\text { - Junk emails } \\
\text { - Terrorism details } \\
\text { - Viruses } \\
\text { - Threats database }\end{array}$ \\
\hline Characteristics & $\begin{array}{l}\text { - Structured } \\
\text { - Customer contents } \\
\text { - Unstructured }\end{array}$ & $\begin{array}{l}\text { - Minimum latency } \\
\text { - High throughput } \\
\text { - Volumes } \\
\text { - Velocity }\end{array}$ & $\begin{array}{l}\text { - Users identity } \\
\text { - Providers profile } \\
\text { - Incomplete file } \\
\text { - Deceptive content } \\
\text { - Confidential codes } \\
\text { - Private network } \\
\text { - Legal information } \\
\text { - Multilingual data }\end{array}$ \\
\hline Analytics & $\begin{array}{l}\text { - Systematic records } \\
\text { - Behavior detection } \\
\text { - Customer care } \\
\text { - Social monitoring } \\
\text { - Controlling text } \\
\text { - Dealing Sentiment }\end{array}$ & $\begin{array}{l}\text { - Scientific theories } \\
\text { - Mathematical laws } \\
\text { - Analytical models }\end{array}$ & $\begin{array}{l}\text { - Crime analysis } \\
\text { - Temporal analysis } \\
\text { - Multilingual text } \\
\text { - Data analytics } \\
\text { - Sentiment analysis } \\
\text { - Crime detections } \\
\text { - Cyber attacks } \\
\text { - Cyber intelligence }\end{array}$ \\
\hline Impact & $\begin{array}{l}\text { - Smart marketing } \\
\text { - User appraisal } \\
\text { - Recommendation } \\
\text { - Customer loyalty } \\
\text { - User satisfaction }\end{array}$ & $\begin{array}{l}\text { - Advanced science } \\
\text { - Smart technologies }\end{array}$ & $\begin{array}{l}\text { - Improved safety } \\
\text { - Efficient security }\end{array}$ \\
\hline
\end{tabular}

solutions influenced with information security should be created to maximize the security zoon around the areas of the big data storage, transmission, and processing. According to Ref. 42, business intelligence and analysis deals with many different analytical approaches which improve the decision making on information security. Also, big data analytics provide us possible situations where big data breaches need to be monitored and when decision making should be delivered. Table 2 summarizes the implication of the big data in the selected use cases of business intelligence and analysis. Further, we can monitor the big data breaches through the applications, data characteristics, analytics techniques, and potential impacts. From Table 2, service providers and users will know how to analyze the big data to make an effective decision which controls the big data breaches in many different situations. 
The data user could take the best decision through the proposed model when the data user handles the big data in public or private environments. Assume that three data users are sending their packets (big data) through our proposed model with three different priorities (high, medium and low respectively). According to the breach calculations which depend on the network calculus algorithm, these three packets are arriving at different times. Here, the best decision is depending on the high priority and the minimum of the delay bound. Based on the data user's best decision, the proposed approach provides the best packet without any data breach might be occurred during the data processing.

\section{Conclusion}

Access control is investigated as an advanced technology of big data analytical techniques which not only helps us to improve the big data security breaches but also decision making based on information security. We deployed the network calculus procedures within the proposed model to monitor the big data breaches through the big data analytical technique. From the results, monitoring services with high priority reduce the delay and therefore it can be selected to improve the solutions. In this research, mitigating big data breaches depend on the decision making which is the final stage of the protection authorized by the service providers.

In the future work, decision making on information security should be automated using the intelligent system while improving unnecessary delay which is important to mitigate the big data security breaches. Although access control security already exists on different platforms, we can develop the adaptable approach which provides the access control security in all situations where platforms are independent. So, we can analyze the time complexity as a future work of this research. To monitor the continuous big data security breaches, we can also introduce the automation techniques.

\section{Acknowledgments}

This project was funded by the Deanship of Scientific Research (DSR) at King Abdulaziz University, Jeddah, Saudi Arabia, under Grant No. 14377-611-549. The authors, therefore, acknowledge with thanks DSR for technical and financial support.

\section{References}

1. U. Sivarajah, M. M. Kamal, Z. Irani and V. Weerakkody, Critical analysis of big data challenges and analytical methods, Journal of Business Research (2016).

2. Z. Yan, W. Ding, X. Yu, H. Zhu and R. H. Deng, Deduplication on encrypted big data in cloud, IEEE Transactions on Big Data 2(2) (2016) 138-150.

3. A. Gandomi and M. Haider, Beyond the hype: Big data concepts, methods, and analytics, International Journal of Information Management 35(2) (2015) 137-144.

4. M. Marchetti, F. Pierazzi, A. Guido and M. Colajanni, May. Countering advanced persistent threats through security intelligence and big data analytics. In 2016 8th International Conference on Cyber Conflict (CyCon), 2016, pp. 243-261, NATO CCD COE. 
5. J. Huang, Y. Sun, Z. Xiong, Q. Duan, Y. Zhao, X. Cao and W. Wang, Modeling and analysis on access control for device-to-device communications in cellular network: A network-calculus-based approach, IEEE Transactions on Vehicular Technology 65(3) (2016) 1615-1626.

6. Verizon Communications Inc. (2013). 2013 Data Breach Investigations Report.

7. http://www.verizonenterprise.com/resources/reports/rp_data-breach-investigationsreport-2013_en_xg.pdf.

8. Y. Yang and X. Zheng, Type based keyword search for securing big data, 2013 International Conference on Cloud Computing and Big Data, IEEE, pp. 354-359.

9. X. Li, F. Zhang and Y. Wang, Research on big data architecture, key technologies, and its measures, 2013 IEEE 11th International Conference on Dependable, Autonomic and Secure Computing, pp. 1-4.

10. J. Hwang and I. Syamsuddin, Information security policy decision making: An analytic hierarchy process approach, in Third Asia International Conference on Modelling and Simulation, 2009, AMS'09., pp. 158-163, IEEE, 2009.

11. J.-J. Peng, J.-Q. Wang and X.-H. Wu, Novel multi-criteria decision-making approaches based on hesitant fuzzy sets and prospect theory, International Journal of Information Technology and Decision Making 15(3) (2016) 621-643.

12. T. Poleto, V. D. Heuer de Carvalho and A. P. C. S. Costa, The full knowledge of big data in the integration of inter-organizational information: An approach focused on decision making, International Journal of Decision Support System Technology 9(1) (2017) 16-31.

13. M. H. Manshaei, Q. Zhu, T. Alpcan, T. Bacşar and J. P. Hubaux, Game theory meets network security and privacy, ACM Computing Surveys (CSUR) 45(3) (2013) 25.

14. L. Xu, C. Jiang, J. Wang, Y. Ren, J. Yuan and M. Guizani, Game theoretic data privacy preservation: Equilibrium and pricing, in 2015 IEEE International Conference on Communications (ICC), pp. 7071-7076, IEEE.

15. A. Acquisti, The economics of personal data and the economics of privacy (2010).

16. L. Xu, C. Jiang, Y. Chen, Y. Ren and K. R. Liu, Privacy or utility in data collection? A contract theoretic approach, IEEE Journal of Selected Topics in Signal Processing $\mathbf{9}(7)$ (2015) 1256-1269.

17. G. Kou, D. Ergu, C. Lin and Y. Chen, Pairwise comparison matrix in multiple criteria decision making, Technological and Economic Development of Economy 22(5) (2016) 738-765.

18. G. Kou, Y. Peng and G. Wang, Evaluation of clustering algorithms for financial risk analysis using MCDM methods, Information Sciences 275 (2014) 1-12.

19. G. Kou, Y. Lu, Y. Peng and Y. Shi, Evaluation of classification algorithms using MCDM and rank correlation, International Journal of Information Technology and Decision Making 11(1) (2012) 197-225.

20. G. Kou and C. Lin, A cosine maximization method for the priority vector derivation in AHP, European Journal of Operational Research 235(1) (2014) 225-232.

21. G. Kou, D. Ergu and J. Shang, Enhancing data consistency in decision matrix: Adapting Hadamard model to mitigate judgment contradiction, European Journal of Operational Research 236(1) (2014) 261-271.

22. Y. Wang, L. Kung and T. A. Byrd, Big data analytics: Understanding its capabilities and potential benefits for healthcare organizations, Technological Forecasting and Social Change (2016), Elsevier.

23. L. Xu, P. D. Khoa, S. H. Kim, W. W. Ro and W. Shi, Another look at secure big data processing: Formal framework and a potential approach, 2015 IEEE 8th International Conference on Cloud Computing, IEEE, 2015, pp. 548-555. 
24. B. Song, M. S. Hossain and A. Alamri, QoS-aware resource provisioning for big data processing in cloud computing environment, 2014 International Conference on Computational Science and Computational Intelligence (CSCI), Vol. 2, IEEE, pp. 107-112.

25. C. Ji, Y. Li, W. Qiu, U. Awada and K. Li, Big data processing in cloud computing environment, 12th International Symposium on Pervasive Systems, Algorithms, and Networks, pp. 17-23, December, 2012.

26. L. Xu, C. Jiang, J. Wang, J. Yuan and Y. Ren, Information security in big data: Privacy and data mining, IEEE Access 2 (2014) 1149-1176.

27. S. Sharma, B. Bhushan and S. Sharma, Improvising information security in cloud computing environment, International Journal of Computer Applications 86(16) (2014) $22-25$.

28. J.-S. Wang, C.-H. Liu and G. T. R. Lin, How to manage information security in cloud computing, 2011 IEEE International Conference on Systems, Man, and Cybernetics (SMC), pp. 1405-1410, 2011.

29. Kumar and H. Lee, Efficient and secure cloud storage for handling big data, in Proceedings of 6th International Conference on New Trends in Information Science and Service Science and Data Mining (ISSDM), October 23-25, 2012, pp. 162-166.

30. X. Zhang, C. Liu, S. Nepal, C. Yang, W. Dou and J. Chen, SaC-FRAPP: A scalable and cost-effective framework for privacy preservation over big data on the cloud, Concurrency and Computation: Practice and Experience, 2013, doi: 10.1002/cpe.3083.

31. R. Zuech, T. M. Khoshgoftaar and R. Wald, Intrusion detection and big heterogeneous data: A survey, Journal of Big Data 2 (2015) 1-41.

32. A. Tankard, Big data security, Network Security 2012(7) (2012) 5-8.

33. N. Gruschka and L. L. Iacono, Vulnerable cloud: Soap message security validation revisited, in IEEE International Conference on Web Services - ICWS 2009, IEEE, 2009, pp. $625-631$.

34. S.-H. Kim, N.-U. Kim and T.-M. Chung, Attribute relationship evaluation methodology for big data security, 2013 International Conference on IT Convergence and Security (ICITCS), IEEE, pp. 1-4.

35. M. Schwartz, New virtualization vulnerability allows escape to hypervisor attacks, Information of Week Security Letter 4 (2012).

36. M. Senthil Kumar, V. Ramasamy, S. Sheen, C. Veeramani, A. Bonato and L. Batten, Computational Intelligence, Cyber Security, and Computational Models (2016).

37. J. Huang, M. Zeng, C. C. Xing, J. Luo and F. Hou, Modeling and analysis for admission control of M2M communications using network calculus, 2017 IEEE International Conference on Communications (ICC), 2017 May 21, pp. 1-6, IEEE.

38. M. Fidler, Survey of deterministic and stochastic service curve models in the network calculus, IEEE Communications Surveys and Tutorials (2010).

39. S. Bondorf and J. B. Schmitt, The DiscoDNC v2 - A comprehensive tool for deterministic network calculus (PDF), 8th International Conference on Performance Evaluation Methodologies and Tools (VALUETOOLS 2014).

40. Y. Long, Z. Lu and H. Shen, Composable worst-case delay bound analysis using network calculus, IEEE Transactions on Computer-Aided Design of Integrated Circuits and Systems (2017).

41. S. Bondorf, May. Better bounds by worse assumptions - Improving network calculus accuracy by adding pessimism to the network model, 2017 IEEE International Conference on Communications (ICC), pp. 1-7, 2017, IEEE.

42. H. Chen, R. H. L. Chiang and V. C. Storey, Business intelligence and analytics: From big data to a big impact. MIS Quarterly, 36(4) (2012) 1165-1188. 